\title{
Screening of COPD patients for abdominal aortic aneurysm
}

Ingo H Flessenkaemper'

Robert Loddenkemper ${ }^{2}$

Stephanie Roll ${ }^{3}$

Kathrin Enke-Melzer'

Henrik Wurps ${ }^{2}$

Torsten T Bauer ${ }^{2}$

'Department for Vascular Medicine, 2Department of Pneumology, Helios Klinikum Emil von Behring, Berlin, Germany; ${ }^{3}$ Institute for Social Medicine, Epidemiology and Health Economics, Charité Universitätsmedizin Berlin, Berlin, Germany
Correspondence: Ingo H Flessenkaemper Department for Vascular Medicine, Helios Klinikum Emil von Behring, Walterhöferstraße, II, I4I65 Berlin, Germany

Tel/fax +49 3081022200

Email ingo.flessenkaemper@helioskliniken.de
This article was published in the following Dove Press journal:

International Journal of COPD

10 June 2015

Number of times this article has been viewed

Purpose: Screening for abdominal aortic aneurysm (AAA) in "men aged over 65 years who have ever smoked" is a recommended policy. To reduce the number of screenings, it may be of value to define subgroups with a higher prevalence of AAA. Since chronic obstructive pulmonary disease (COPD) and AAA are associated with several common risk factors, this study investigates the prevalence of AAA in COPD patients.

Patients and methods: Patients with COPD were identified via the hospital information system. Inclusion criteria were: COPD stage I-IV, ability to give full consent, and age $>18$ years; exclusion criteria were: patient too obese for an ultrasound check, previously diagnosed AAA, prior surgery for AAA, or ethical grounds such as concomitant advanced malignant or endstage disease. The primary endpoint of the study was an aortic diameter measured by ultrasound of $\geq 30 \mathrm{~mm}$. Defined secondary endpoints were evaluated on the basis of medical records and interviews.

Results: Of the 1,180 identified COPD patients, 589 were included in this prospective study. In 22 patients $(3.70 \%$ ), the aortic diameter was $\geq 30 \mathrm{~mm}$, representing an AAA prevalence of $6.72 \%$ among males aged $>65$ years. The risk of AAA increased with the following comorbidities/risk factors: male sex (odds ratio [OR] 2.98), coronary heart disease (OR 2.81), peripheral arterial occlusive disease (OR 2.47), hyperlipoproteinemia (OR 2.77), AAA in the family history (OR 3.95), and COPD stage I/II versus IV (OR 1.81).

Conclusion: The overall AAA prevalence of $3.7 \%$ in our group of COPD patients is similar to that of the general population aged $>65$ years. However, the frequency of AAA in male COPD patients aged $>65$ years is considerably higher $(6.72 \%)$ and increased further still in those individuals with additional comorbidities/risk factors. Defining subgroups with a higher risk of AAA may increase the efficiency of screening.

Keywords: chronic obstructive pulmonary disease, AAA, screening, risk factors, epidemiology, inflammatory disease, tobacco abuse

\section{Introduction}

In the US, ${ }^{1}$ the UK, ${ }^{2,3}$ Denmark, ${ }^{4}$ and Western Australia, ${ }^{5}$ recommendations for a population-based screening program for abdominal aortic aneurysms (AAA) exist, as the general benefits of this approach have been proven. ${ }^{6-10}$ To date, such programs have been realized in the US, England, Sweden, Scotland, Northern Ireland, and Norway. ${ }^{11}$ However, it seems useful to reduce the number of patients to be screened by identifying groups at a higher risk of developing AAA. In a previous study, we found an elevated AAA prevalence of $8.6 \%$ in patients who had been treated for arterial disease previously. ${ }^{12}$ Whether other diseases are also related to AAA remains unclear. The US Preventive Services Task Force (USPSTF) defined the criteria for the identification of persons who should be screened. The main group to screen includes "men aged over 65 years who ever smoked". ${ }^{13,14}$ It seems meaningful to look for additional 
conditions that may be associated with a higher prevalence of AAA. While a higher prevalence of cardiovascular disease in chronic obstructive pulmonary disease (COPD) patients has been shown, ${ }^{15}$ the figures for AAA in COPD patients are unclear. Therefore, the objective of the present study is to estimate the proportion of hospitalized patients with COPD who are also afflicted by AAA.

\section{Patients and methods}

We performed a prospective cross-sectional study in our hospital. All pulmonary department inpatients with a COPD diagnosis were identified via the hospital information system. The study was approved by the ethics committee of the Charité - Universitätsmedizin Berlin (reg nr EA4/142/13). All aspects of the study were in accordance with the Declaration of Helsinki (in its current, revised form). A study nurse assessed the identified patients for inclusion and exclusion criteria. Inclusion criteria were: COPD stage I-IV, ${ }^{16}$ minimum age of 18 years, and ability to give full consent. Patients too obese for an ultrasound check on technical grounds, those with previously diagnosed AAA, and patients who had already undergone surgery for AAA were excluded, as were some patients on ethical grounds (see "Results").

If patients met the inclusion criteria, the study nurse contacted the responsible physician for further information about the patient and his/her circumstances, in order to avoid contacting patients in a situation not appropriate for discussing a study (eg, concomitant advanced malignant or end-stage disease).

Once the treating medical team had established that the patient was in an appropriate situation and had agreed to the patient being contacted, the patient was informed about the study. After obtaining the patient's oral and written consent, the following factors were assessed in a standardized interview: smoking status, hypertension, coronary heart disease (CHD), myocardial infarction, peripheral arterial occlusive disease (PAOD), stroke, diabetes, hyperlipoproteinemia (HLP), pulmonary embolism, thrombosis, hyperuricemia, malignancy, abdominal symptoms, and the family history on AAA. Respiratory and other data were extracted from the patient's record.

Ultrasound screening for AAA was performed by a study nurse (trained on AortaScan ${ }^{\circledR}$ AMI 9700; Verathon, Bothell, WA, USA). AAA was defined as an aortic diameter $\geq 30 \mathrm{~mm}$. Where an aortic diameter $\geq 30 \mathrm{~mm}$ was found or in cases of ambiguous findings, an angiologist was involved.

Patient characteristics are presented as frequencies and percentages for categorical data, and as means with standard deviations (SDs) or medians with ranges for continuous data. For the analysis, COPD stages I and II were combined. Associations between AAA and suspected risk factors were evaluated by univariate logistic regression; results are presented as odds ratios (ORs) with $95 \%$ confidence intervals (CIs) and $P$-values, which are considered explorative (without adjusting for multiple testing). Analyses were performed in SAS version 9.2 (SAS Institute, Cary, NC, USA).

\section{Results}

In a 24-month period (February 2011-February 2013), 1,481 patients with COPD were identified via the clinical information system. Of these, 301 patients were excluded because of readmission to the hospital. A total of 1,180 patients thus qualified for the study, of whom 589 agreed to participate and 591 were excluded. Patients were excluded either because they had already received surgery for AAA (13 patients); or on ethical grounds, such as concomitant advanced malignant or end-stage disease (286 patients); or because they did not agree to participate in the study (125 patients). For linguistic reasons, 29 patients were not able to comply with the requirements for giving informed consent and 87 patients had left the hospital prior to being contacted. In 51 patients, obesity rendered an ultrasound check technically impossible (Figure 1).

The study participants were Caucasian, had a mean age of approximately 70 years (only eight patients below 50 years), a mean body mass index (BMI) of $25.4 \mathrm{~kg} / \mathrm{m}^{2}$, and $54.2 \%$ were males. The average tobacco consumption was 50.2 pack-years. C-reactive protein (CRP) was elevated on average $(26.8 \mathrm{mg} / \mathrm{L}$; normal value $<5 \mathrm{mg} / \mathrm{L})$. More than half $(56 \%)$ of the patients were COPD stage IV, with mean forced expiratory volume in 1 second $\left(\mathrm{FEV}_{1}\right)$ values of $\mathrm{FEV}_{1} 0.9 \mathrm{~L}$ and $\mathrm{FEV}_{1}$ predicted (pred) $36.8 \%$; maximum vital capacity $\left(\mathrm{VC}_{\max }\right)$ values of $\mathrm{VC}_{\max } 2.01$ and $\mathrm{VC}_{\max }$ pred $57.8 \%$, and $\mathrm{FEV}_{1} / \mathrm{VC}_{\max } 43.2 \%$ (Table 1 ).

Table 2 shows the frequency of comorbidities and potential confounding factors in these COPD patients. Patients were predominately current or former smokers $(n=546$, 92.9\%) and had additional comorbidities, such as hypertension $(n=369,63.1 \%)$, CHD $(n=139,23.7 \%)$, myocardial infarction $(n=78,13.3 \%)$, PAOD $(n=65,11.1 \%)$, and stroke $(n=44,7.5 \%)$. At least one kind of arterial disease was suffered by 210 patients (35.7\%). Overall, 109 patients (18.6\%) had one arterial disease, 87 patients $(14.8 \%)$ had two, 13 patients $(2.2 \%)$ had three, and one patient $(0.2 \%)$ suffered from four arterial diseases.

In terms of other comorbidities, 141 patients $(24 \%)$ had diabetes, 59 (10\%) had HLP, and 127 patients (22\%) had 


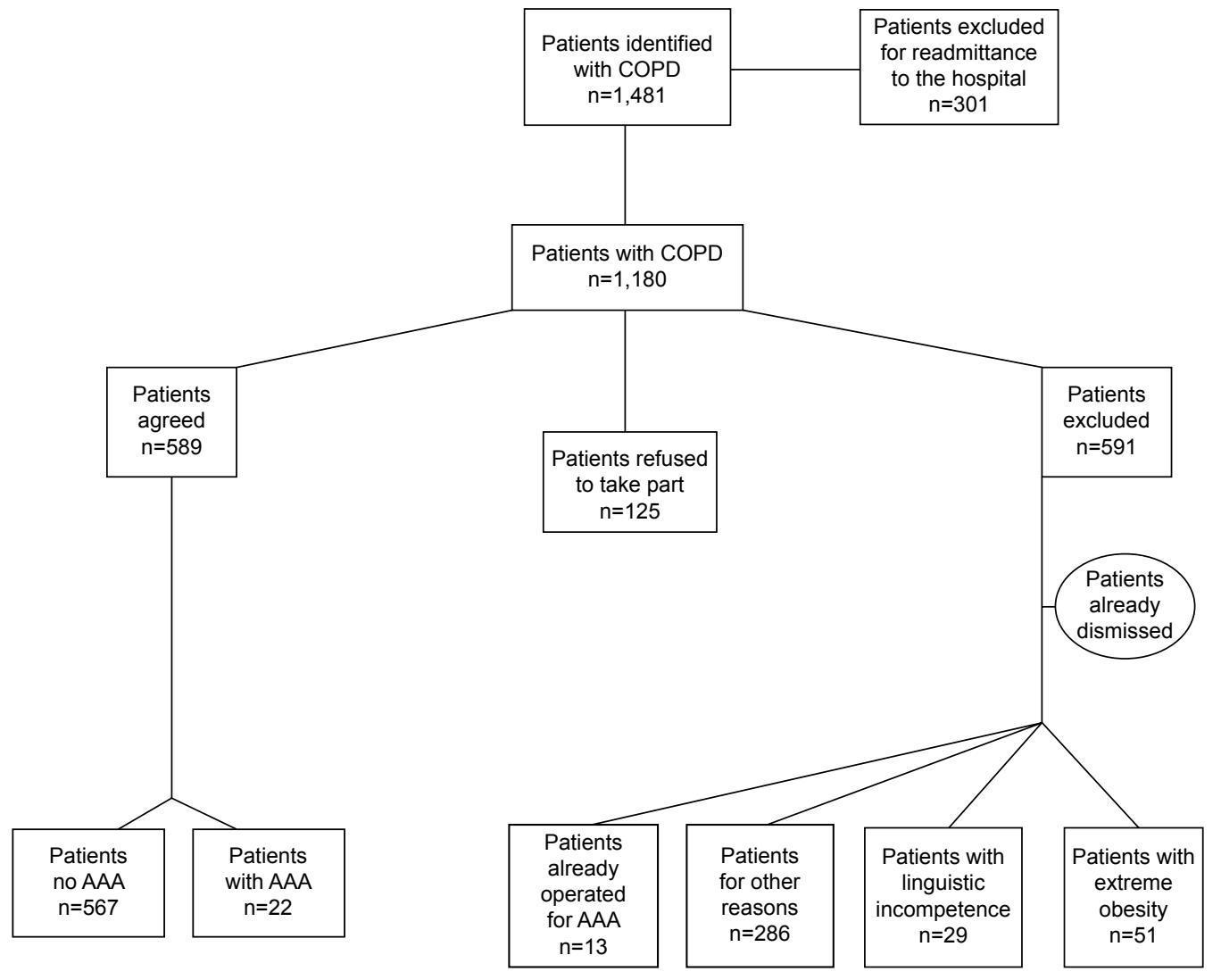

Figure I Study population flowchart.

Abbreviations: COPD, chronic obstructive pulmonary disease; AAA, abdominal aortic aneurysm.

Table I Basic characteristics and pulmonary function

\begin{tabular}{|c|c|}
\hline & Mean \pm SD or $\mathbf{n}(\%)$ \\
\hline Age (years) & $70.2 \pm 9.1$ \\
\hline Male & $319(54.2)$ \\
\hline Female & $270(45.8)$ \\
\hline BMI $\left(\mathrm{kg} / \mathrm{m}^{2}\right)$ & $25.4 \pm 6.2$ \\
\hline Package years & $50.2 \pm 36.1$ \\
\hline CRP (mg/L) & $26.8 \pm 48.8$ \\
\hline \multicolumn{2}{|l|}{ Stage of COPD } \\
\hline$I+I I$ & I 33 (23.5) \\
\hline III & I I8 (20.8) \\
\hline \multirow[t]{2}{*}{ IV } & $315(55.7)$ \\
\hline & $\begin{array}{l}\text { Mean } \pm \text { SD } \\
\text { median (range) }\end{array}$ \\
\hline \multirow[t]{2}{*}{$\mathrm{FEV}_{1} \mathrm{~L}$} & $0.9 \pm 0.6$ \\
\hline & $0.9(0.31-2.8)$ \\
\hline \multirow[t]{2}{*}{$\mathrm{FEV}_{1} \%$ pred } & $36.8 \pm 20.6$ \\
\hline & $35.7(11.4-108.1)$ \\
\hline \multirow[t]{2}{*}{$V C_{\max } \mathrm{L}$} & $2.0 \pm 2.5$ \\
\hline & $2.0(0.62-5.12)$ \\
\hline \multirow[t]{2}{*}{$\mathrm{VC}_{\max } \%$ pred } & $57.8 \pm 27.4$ \\
\hline & $60.3(18.3-139.7)$ \\
\hline $\mathrm{FEV}_{1}$ & $43.2 \pm 19.5$ \\
\hline$V C_{\max }$ & $45.0(16-86.0)$ \\
\hline
\end{tabular}

Abbreviations: SD, standard deviation; BMI, body mass index; CRP, C-reactive protein; COPD, chronic obstructive pulmonary disease; $\mathrm{FEV}_{1}$, forced expiratory volume in I second; pred, predicted; VC, vital capacity; max, maximum. malignancies, of which 91 (72\%) were in the lower respiratory tract (mainly lung cancer). Abdominal symptoms were experienced by 43 patients $(7.3 \%)$, and $16(2.7 \%)$ knew of AAA in their families (Table 2).

Among the 589 patients, $22(3.7 \%)$ had an AAA (aortic diameter $\geq 30 \mathrm{~mm}$; Table 3$)$. Of these 22 patients, $17(77.0 \%)$ were males, $20(91.0 \%)$ were current or former smokers, 14 $(63.0 \%)$ had hypertension, ten $(42.0 \%)$ suffered from CHD, four $(18.0 \%)$ had already had a myocardial infarction, five $(23.0 \%)$ suffered from PAOD, three $(13.6 \%)$ had experienced a stroke, four (18.0\%) had diabetes, five $(23.0 \%)$ had HLP, and eight (36.4\%) had a malignancy. AAA diameters are summarized in Table 4; in most cases, the diameter was between $30 \mathrm{~mm}$ and $45 \mathrm{~mm}$. The prevalence of AAA was $5.3 \%$ among males and only $1.9 \%$ among females. The prevalence rates of AAA among patients aged $>65$ years were $6.72 \%$ and $2.54 \%$ for males and females, respectively.

Of all patients with malignancies, $6.3 \%$ were afflicted by AAA. In the case of AAA in the family history, $9.0 \%$ of patients had an AAA themselves. Individuals with HLP had an AAA in $8.5 \%$ of cases, but only $2.8 \%$ of patients with diabetes had an AAA. Of patients with PAOD, 5.3\% were 
Table 2 Comorbidities and potential contributory factors $(n=589)$

\begin{tabular}{lll}
\hline & $\mathbf{n}$ & $\%$ \\
\hline Smoker & & \\
$\quad$ Former & 388 & 66.0 \\
$\quad$ Current & 158 & 26.9 \\
$\quad$ Never & 41 & 7.3 \\
Hypertension & 369 & 63.1 \\
Coronary heart disease & 139 & 23.7 \\
Myocardial infarction & 78 & 13.3 \\
Peripheral arterial occlusive disease & 65 & 11.1 \\
Stroke & 44 & 7.5 \\
All kinds of arterial diseases & 210 & 35.7 \\
Diabetes mellitus & 141 & 24.1 \\
Hyperlipoproteinemia & 59 & 10.1 \\
Pulmonary embolism & 26 & 4.4 \\
Deep vein thrombosis & 27 & 4.6 \\
Hyperuricemia & 3 & 0.5 \\
Abdominal pain & 43 & 7.3 \\
Malignancy & 127 & 21.6 \\
Malignancies of the lower respiratory tract & 91 & 15.4 \\
Family history of abdominal aortic aneurysm & 16 & 2.7 \\
\hline
\end{tabular}

identified as carrying an AAA, as were $6.1 \%$ of CHD patients, $2.8 \%$ of patients with a previous myocardial infarction, and $6.8 \%$ of patients with a previous stroke. Of the patients complaining of abdominal symptoms, only $2.5 \%$ had an AAA. The proportion of patients with thrombosis, pulmonary embolism, hypertension, or current or former tobacco consumption with AAA in this subgroup ranged between $3.4 \%$ and $3.8 \%$, and thus did not differ from the overall median values. COPD in stage I or II was associated with AAA in $4.5 \%$ of cases, COPD in stage III in $4.2 \%$, and COPD in stage IV was associated with AAA in $2.5 \%$ of patients.

In addition, we looked for further patient data and parameters in the patient's history that might possibly be associated with a higher prevalence of AAA. Besides age (OR 1.08 per year, $P$-value $0.004,95 \%$ CI 1.03-1.14) and male sex (OR 3.0, $P$-value 0.034, 95\% CI 1.1-8.2), which were associated with a higher prevalence of AAA; further

Table 3 Comorbidities of patients with AAA ( $\geq 30 \mathrm{~mm} ; \mathrm{n}=22$ )

\begin{tabular}{lll}
\hline Comorbidity & $\mathbf{n}$ & $\%$ \\
\hline Male & 17 & 77 \\
Current or former smoker & 20 & 91 \\
Hypertension & 14 & 63 \\
Coronary heart disease & 10 & 42 \\
Myocardial infarction & 4 & 18 \\
Peripheral arterial occlusive disease & 5 & 23 \\
Stroke & 3 & 14 \\
Diabetes & 4 & 18 \\
Hyperlipoproteinemia & 5 & 23 \\
Malignancy & 8 & 36 \\
\hline
\end{tabular}

Abbreviation: AAA, abdominal aortic aneurysm.
Table 4 Diameter of identified AAA ( $\geq 30 \mathrm{~mm})$

\begin{tabular}{ll}
\hline Diameter $(\mathbf{m m})$ & Number of patients \\
\hline $30-35$ & 5 \\
$36-40$ & 4 \\
$4 I-45$ & 5 \\
$46-50$ & 1 \\
$5 I-60$ & 1 \\
$6 I-70$ & 3 \\
$>70$ & 2 \\
Not specified & 1 \\
Total & 22 \\
\hline
\end{tabular}

Abbreviation: AAA, abdominal aortic aneurysm.

significant associations were found between CHD (OR 2.8, $P$-value $0.019,95 \% \mathrm{CI} 1.2-6.7)$ and the presence of any arterial disease (OR 2.7, $P$-value $0.025,95 \%$ CI $1.13-6.42$ ) and AAA. The presence of PAOD alone (OR 2.47, $P$-value $0.086,95 \%$ CI $0.88-6.94$ ) or HLP (OR 2.8, $P$-value 0.054 , $95 \%$ CI 0.98-7.81) also showed an increased risk; however, these effects did not reach statistical significance. Factors tested but not significantly associated with AAA were: BMI (OR 1.02, $P$-value $0.468,95 \%$ CI 0.96-1.09), cigarette packyears (OR 1.00, $P$-value $0.580,95 \%$ CI $0.99-1.01$ ), and CRP (OR 1.00, $P$-value 0.166, 95\% CI 1.00-1.01; Figure 2).

\section{Discussion}

This prospective study of 589 COPD patients showed a prevalence of $3.7 \%$ for AAA defined as an aortic diameter $\geq 30 \mathrm{~mm}$. This prevalence of AAA among COPD patients is similar to that seen in screening studies of the general population, ie, those not limited to COPD patients (eg, the multicenter aneurysm screening study [MASS] 4.9\%, ${ }^{3}$ Lindholt et al $\left.4.0 \%{ }^{3,4}\right)$. If the calculation is based on the same criterion as in the quoted general screening studies - ie, men aged $>65$ years - the prevalence of $6.72 \%$ is considerably higher and very close to the rate of $6.5 \%$ recently cited by Divo et al in an overview on COPD comorbidities. ${ }^{17,18}$ Therefore, an amendment to the US recommendations for AAA screening would generate a higher prevalence of AAA if COPD patients were to be defined as a subgroup among "men aged $>65$ years who have ever smoked" who are particularly worth screening. In addition, some other subgroups of COPD patients with an even higher prevalence of AAA were identified (CHD, HLP, malignoma, positive family history for AAA). This is in accordance with the literature, where various studies showing, eg, a CHD-related prevalence of AAA ranging from $9.0 \%-14.4 \%$ were found. ${ }^{19-22}$ A highly significant risk factor was sex. To be a male with COPD means having a three-fold higher risk of developing an AAA. 


Parameter
Male vs female
Ex smoker
Smoker
Hypertension
CHD
Myocardial infarction
PAOD
Stroke
Diabetes mellitus
Hyperlipoproteinemia
Deep venous thrombosis
Pulmonary embolism
Abdominal pain
Malignancy
Family history of AAA
COPD stage I or II vs IV
COPD stage III vs IV
Any arterial disease
Estimate $(95 \% \mathrm{Cl})$

$2.983(1.086,8.197)$ $1.649(0.212,12.804)$ $1.340(0.152,11.788)$ $1.025(0.423,2.485)$ $2.810(1.187,6.653)$ $1.471(0.485,4.468)$ $2.471(0.880,6.937)$ $2.010(0.571,7.076)$ $0.693(0.230,2.081)$ $2.772(0.984,7.811)$

$0.984(0.127,7.596)$

$1.025(0.132,7.926)$

$1.297(0.293,5.754)$

$2.151(0.882,5.248)$

$3.950(0.841,18.560)$

$1.813(0.617,5.331)$

$1.698(0.544,5.298)$

$2.698(1.133,6.423)$

AAA/y
$17 / 319$
$15 / 388$
$5 / 158$
$14 / 369$
$10 / 139$
$4 / 78$
$5 / 65$
$3 / 44$
$4 / 141$
$5 / 59$
$1 / 27$
$1 / 26$
$2 / 41$
$8 / 127$
$2 / 16$
$6 / 133$
$5 / 118$
$13 / 210$

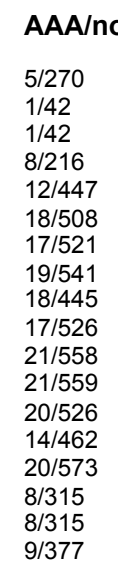

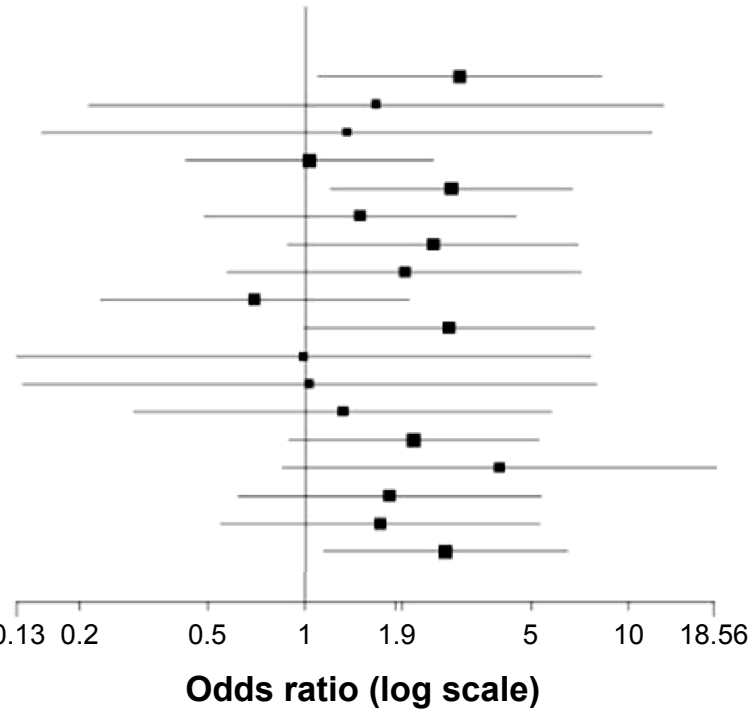

Figure 2 Comorbidities and risk factors in COPD.

Note: COPD patients and the likelihood of finding an AAA expressed as odds ratios.

Abbreviations: CHD, coronary heart disease; PAOD, peripheral arterial occlusive disease; AAA, abdominal aortic aneurysm; COPD, chronic obstructive pulmonary disease; $\mathrm{Cl}$, confidence interval; vs, versus.

In contrast to the situation in males, a Cochrane Review for screening in women found that 3-5 years after screening, women had an OR 1.99 (95\% CI 0.36-10.88; versus men OR $0.60,95 \%$ CI $0.47-0.78$ ) as compared to an unscreened population. This means that more women died in the screened population, thus the frequency of ruptured AAA could not be reduced by screening in women. ${ }^{23}$ The same result was found by Scott et al. ${ }^{24}$ The results of a recent Swedish study suggest screening subgroups, such as women older than 70 years who have ever smoked. ${ }^{25}$ It thus seems more relevant to develop a screening program that includes more risk factors than just age, sex, and one major disease like COPD. The combination of the above-mentioned risk factors might help to develop screening strategies that substantially reduce the number of necessary screenings.

In addition to the 22 patients in our study in whom AAA was detected, 13 (3.73\%) of the 591 excluded patients had received prior surgery for AAA, suggesting that within the group of excluded patients, AAAs are also present. Of the 22 patients with an aortic diameter $\geq 30 \mathrm{~mm}$, six (27.0\%) had passed the limit of an AAA diameter of $50 \mathrm{~mm}$ at which therapeutic options have to be considered. An urgent indication for considering therapy was detected in four patients.

The observed comorbidities are typical for a population with a high prevalence of smokers or ex-smokers with a relatively high average of 50.2 pack-years. The nicotine load was so high in this population that no difference could be observed for AAA patients. Overall, 65 patients (11.0\%) were already afflicted by PAOD, which shows the importance of also screening COPD patients for this disease, as well as for other vascular diseases like CHD (23.7\%). These anamnestic hints agree with reports in the literature about a higher prevalence of AAA in patients with $\mathrm{CHD}^{19-22}$ or noncardiac arterial disease. ${ }^{12,26}$

The Lung Health Study has shown a close relationship between COPD and an increased risk of cardiovascular disease and death. ${ }^{27}$ This was confirmed by a recently published investigation in which $65 \%$ of men and $55 \%$ of women with COPD were afflicted by cardiovascular disease. In the latter study, cardiovascular diseases (all grouped together, ie, not separated into subgroups) were not a factor contributing to the reduction in COPD patients' health-related quality of life. ${ }^{28}$ Inflammation seems to play a major common role in COPD and atherosclerosis. ${ }^{29} \mathrm{~A}$ study on the association of aortic aneurysms and reduced lung function with risk factors such as cigarette smoking, cardiovascular disease, and increased inflammatory and hemostatic activity found that individuals with AAA had a higher frequency of impaired lung function and a greater incidence of COPD compared to controls without an aneurysm. ${ }^{30}$ In this study, the markers of activated inflammation and hemostasis had the most important effect. The main factors for the identification of an activation of inflammation and hemostasis were plasmin-antiplasmin complexes and D-dimers. Although impaired lung functiondetermined by $\mathrm{FEV}_{1}, \mathrm{FEV}_{1}$ /forced vital capacity (FVC), and peak expiratory flow rate (PEFR) - could be demonstrated in aneurysm patients compared to a control group with PAOD, cigarette smoking and cardiovascular disease in COPD 
patients were not as strongly related to aneurysm formation as these markers of inflammation. The authors hypothesized that activation of inflammation and hemostasis in response to injury may be an important explanation for the association between aneurysm formation and reduced respiratory function. The higher incidence of COPD in aneurysm patients was shown in a Dutch case-control study, where the $\mathrm{FEV}_{1} / \mathrm{FVC}$ ratio was reduced in an AAA group versus PAOD patients. This was apparent in current, former, and never smokers; the largest difference was found for the latter group. The authors concluded that the pronounced airway obstruction cannot be explained by cigarette smoking alone. They suggest that other factors in addition to smoking are responsible for the association between COPD and AAA. ${ }^{31}$ Their analysis of the factors underlying development of COPD and AAA is in accordance with the results of an earlier study, where the largest difference in the $\mathrm{FEV}_{1} / \mathrm{FVC}$ ratio was also found in the group of never smokers. ${ }^{32}$ This association could not be assessed in the present study, due to the lack of a sufficiently large control group of never smokers.

Smoking is not only a risk factor for AAA, but also for CHD. However, the relationship between impaired lung function and AAA is tighter than the relationship between decreased lung function and CHD. ${ }^{31,32}$ The question is, whether both pathologies are part of a process that Fabbri and Rabe call "chronic systemic inflammatory syndrome", 33 a generalized inflammatory process which they assume is responsible for various clinical entities. This inflammation could cause COPD in the lung and AAA in the vascular system. Inflammatory factors such as IL-6, IL1- $\beta$, TNF- $\alpha$, MMP-9, MCP-1, and high-sensitivity CRP are elevated in COPD patients, ${ }^{29}$ these factors are known to be elevated in AAA patients as well. Reports that statins, with their antiinflammatory activity, might have a beneficial role in COPD treatment also fit into this pattern. ${ }^{34,35}$ Statins are known to suppress inflammatory activity in the wall of AAA, although it has not yet been proven that AAA growth can be retarded by statin medication. ${ }^{36-38}$

In our cohort, an activated level of inflammation with a mean CRP of $26.81 \mathrm{mg} / \mathrm{L}$ was found, which would be consistent with the hypothesis of general inflammation, but is certainly not proof of it. We did not find a statistically significant relationship between CRP level and an increased risk of AAA.

Our findings cannot contribute to solving the problem of whether there is a generalized state of inflammation that initiates different clinical diseases or whether one of these diseases leads to an inflammation that subsequently causes other clinical manifestations. Only the third option, ie, that these comorbidities exist independently of each other, seems to be excluded by both our findings and other reports in the literature revealing strong epidemiologic evidence for reduced $\mathrm{FEV}_{1}$ being a marker for cardiovascular mortality, independent of smoking history. ${ }^{39}$

One limitation of the present study is that out of 1,180 screened COPD patients, only 589 could be included. Therefore, selection bias might be present and may have resulted in a deviation from the "true" prevalence of AAA in this population. However, in half of the excluded patients, the grounds for their noninclusion were ethical. Because these patients are unlikely to be screened in a real-life setting, this patient selection should not lower the practical importance of our findings. A further limitation could be the inclusion of hospital inpatients only, who might have more severe comorbidities than outpatients with COPD. The current study group contained twice as many COPD patients with diabetes, almost four-times as many patients with CHD, and approximately 12-times as many individuals with malignancies as COPD patients observed by general practitioners. ${ }^{15}$

\section{Conclusion}

The prevalence of AAA in a group of hospitalized COPD patients is similar to that of the general population aged over 65 years. However, identifying risk factors such as male sex and CHD may significantly increase the efficiency of screening for AAA. Other factors, such as HLP, PAOD, malignancy, and/or a positive family history for AAA also increased the risk for AAA, but failed to reach statistical significance.

\section{Disclosure}

The authors report no conflicts of interest in this study.

\section{References}

1. U.S. Preventive Services Task Force. Screening for abdominal aortic aneurysm: recommendation statement. Ann Intern Med. 2005;142(3): 198-202.

2. Scott RA, Tisi PV, Ashton HA, Allen DR. Abdominal aortic aneurysm rupture rates: a 7-year follow-up of the entire abdominal aortic aneurysm population detected by screening. J Vasc Surg. 1998;28(1): 124-128.

3. Multicentre Aneurysm Screening Study Group. Multicentre aneurysm screening study (MASS): cost effectiveness analysis of screening for abdominal aortic aneurysms based on four year results from randomised controlled trial. BMJ. 2002;325(7373):1135-1142.

4. Lindholt JS, Juul S, Fasting H, Henneberg EW. Screening for abdominal aortic aneurysms: single centre randomised controlled trial. $B M J$. 2005;330(7494):750.

5. Norman PE, Jamrozik K, Lawrence-Brown MM, et al. Population based randomised controlled trial on impact of screening on mortality from abdominal aortic aneurysm. BMJ. 2004;329(7477):1259-1265. 
6. Dabare D, Lo TT, McCormack DJ, Kung VW. What is the role of screening in the management of abdominal aortic aneurysms? Interact Cardiovasc Thorac Surg. 2012;14(4):399-405.

7. Böckler D, Lang W, Debus ES, et al. Randomisierte studien mit EBM-level 1 beweisen es: ein screening programm für abdominelle aortenaneurysmen ist sinnvoll! [Randomised studies with EBM level 1 prove it. A screening programme for abdominal aortic aneurysms makes sense]. Gefässchirurgie. 2009;14:350-361.

8. Eckstein HH, Bockler D, Flessenkamper I, Schmitz-Rixen T, Debus S, Lang W. Ultrasonographic screening for the detection of abdominal aortic aneurysms. Dtsch Arztebl Int. 2009;106(41):657-663.

9. Ferket BS, Grootenboer N, Colkesen EB, et al. Systematic review of guidelines on abdominal aortic aneurysm screening. J Vasc Surg. 2012;55(5):1296-1304.

10. Moll FL, Powell JT, Fraedrich G, et al; European Society for Vascular Surgery. Management of abdominal aortic aneurysms clinical practice guidelines of the European society for vascular surgery. Eur J Vasc Endovasc Surg. 2011;41(suppl 1):S1-S58

11. Stather PW, Sidloff DA, Rhema IA, Choke E, Bown MJ, Sayers RD. A review of current reporting of abdominal aortic aneurysm mortality and prevalence in the literature. Eur J Vasc Endovasc Surg. 2014; 47(3):240-242.

12. Flessenkamper I, Kendzia A, Stalke J. Multizentrisches Screening eines arteriell vorerkrankten patientenkollektivs in hinblick auf die prävalenz infrarenaler aortenaneurysmen. BARE - Berliner aeurysma raten evaluation. [Multicenter aortic aneurysm screening trial in an arterial sick cohort. BARE - Berlin aneurysm rate evaluation]. Gefaesschirurgie. 2009;14(5):376-383.

13. Fleming C, Whitlock EP, Beil TL, Lederle FA. Screening for abdominal aortic aneurysm: a best-evidence systematic review for the U.S. preventive services task force. Ann Intern Med. 2005;142(3):203-211.

14. Guirguis-Blake JM, Beil TL, Senger CA, Whitlock EP. Ultrasonography screening for abdominal aortic aneurysms: a systematic evidence review for the U.S. preventive services task force. Ann Intern Med. 2014 160(5):321-329.

15. Cazzola M, Bettoncelli G, Sessa E, Cricelli C, Biscione G. Prevalence of comorbidities in patients with chronic obstructive pulmonary disease. Respiration. 2010;80(2):112-119.

16. Bakke PS, Ronmark E, Eagan T, et al; European Respiratory Society Task Force. Recommendations for epidemiological studies on COPD. Eur Respir J. 2011;38(6):1261-1277.

17. Schnell K, Weiss CO, Lee T, et al. The prevalence of clinically-relevant comorbid conditions in patients with physician-diagnosed COPD: a cross-sectional study using data from NHANES 1999-2008. BMC Pulm Med. 2012;12:26.

18. Divo MJ, Martinez CH, Mannino DM. Ageing and the epidemiology of multimorbidity. Eur Respir J. 2014;44(4):1055-1068.

19. Dall'Olmo C, Ippolito A, McIlduff J, Kinning W, Fortin G, et al. EPics I study: evaluation of possible abdominal aortic aneurysms (in patients who have undergone previous CABG). Vasc Dis Manag. 2007; 4(1): $1-6$

20. Durieux R, van Damme H, Labropoulos N, et al. High prevalence of abdominal aortic aneurysm in patients with three-vessel coronary artery disease. Eur J Vasc Endovasc Surg. 2014;47(3):273-278.
21. Long A, Bui HT, Barbe C, et al. Prevalence of abdominal aortic aneurysm and large infrarenal aorta in patients with acute coronary syndrome and proven coronary stenosis: a prospective monocenter study. Ann Vasc Surg. 2010;24(5):602-608.

22. Monney P, Hayoz D, Tinguely F, et al. High prevalence of unsuspected abdominal aortic aneurysms in patients hospitalised for surgical coronary revascularisation. Eur J Cardiothorac Surg. 2004;25(1):65-68.

23. Cosford PA, Leng GC. Screening for abdominal aortic aneurysm. Cochrane Database Syst Rev. 2007;2:CD002945.

24. Scott RA, Bridgewater SG, Ashton HA. Randomized clinical trial of screening for abdominal aortic aneurysm in women. Br J Surg. 2002;89(3):283-285.

25. Svensjo S, Bjorck M, Wanhainen A. Current prevalence of abdominal aortic aneurysm in 70-year-old women. Br J Surg. 2013;100(3):367-372.

26. Kent KC, Zwolak RM, Egorova NN, et al. Analysis of risk factors for abdominal aortic aneurysm in a cohort of more than 3 million individuals. J Vasc Surg. 2010;52(3):539-548.

27. Anthonisen NR, Connett JE, Enright PL, Manfreda J. Hospitalizations and mortality in the lung health study. Am J Respir Crit Care Med. 2002; 166(3):333-339.

28. Sundh J, Johansson G, Larsson K, et al. Comorbidity and health-related quality of life in patients with severe chronic obstructive pulmonary disease attending Swedish secondary care units. Int J Chron Obstruct Pulmon Dis. 2015;10:173-183.

29. Han MK, McLaughlin VV, Criner GJ, Martinez FJ. Pulmonary diseases and the heart. Circulation. 2007;116(25):2992-3005.

30. Fowkes FG, Anandan CL, Lee AJ, et al. Reduced lung function in patients with abdominal aortic aneurysm is associated with activation of inflammation and hemostasis, not smoking or cardiovascular disease. J Vasc Surg. 2006;43(3):474-480.

31. Meijer CA, Kokje VB, van Tongeren RB, et al. An association between chronic obstructive pulmonary disease and abdominal aortic aneurysm beyond smoking: results from a case-control study. Eur J Vasc Endovasc Surg. 2012;44(2):153-157.

32. Sakamaki F, Oya H, Nagaya N, Kyotani S, Satoh T, Nakanishi N. Higher prevalence of obstructive airway disease in patients with thoracic or abdominal aortic aneurysm. J Vasc Surg. 2002;36(1):35-40.

33. Fabbri LM, Rabe KF. From COPD to chronic systemic inflammatory syndrome? Lancet. 2007;370(9589):797-799.

34. Janda S, Park K, FitzGerald JM, Etminan M, Swiston J. Statins in COPD: a systematic review. Chest. 2009;136(3):734-743.

35. Soyseth V, Brekke PH, Smith P, Omland T. Statin use is associated with reduced mortality in COPD. Eur Respir J. 2007;29(2):279-283.

36. Assar AN. Medical treatment of small abdominal aortic aneurysm. J Cardiovasc Surg (Torino). 2012;53(4):517-525.

37. Rughani G, Robertson L, Clarke M. Medical treatment for small abdominal aortic aneurysms. Cochrane Database Syst Rev. 2012;9: CD009536.

38. van der Meij E, Koning GG, Vriens PW, et al. A clinical evaluation of statin pleiotropy: statins selectively and dose-dependently reduce vascular inflammation. PLoS One. 2013;8(1):e53882.

39. Sin DD, Wu L, Man SF. The relationship between reduced lung function and cardiovascular mortality: a population-based study and a systematic review of the literature. Chest. 2005;127(6):1952-1959.

International Journal of COPD

\section{Publish your work in this journal}

The International Journal of COPD is an international, peer-reviewed journal of therapeutics and pharmacology focusing on concise rapid reporting of clinical studies and reviews in COPD. Special focus is given to the pathophysiological processes underlying the disease, intervention programs, patient focused education, and self management protocols.

This journal is indexed on PubMed Central, MedLine and CAS. The manuscript management system is completely online and includes a very quick and fair peer-review system, which is all easy to use. Visit http://www.dovepress.com/testimonials.php to read real quotes from published authors. 